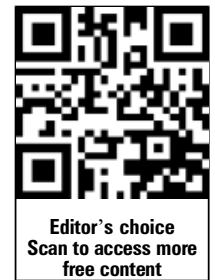

free content
${ }^{1}$ Centre for Sports and Exercise Science, School of Biological Sciences, University of Essex, Colchester, Essex, UK ${ }^{2}$ Department of Physiotherapy, TEl of Lamia, Greece

Correspondence to Dr Gavin R H Sandercock, Centre for Sports and Exercise Science, School of Biological Sciences, University of Essex, Wivenhoe Park, Colchester, Essex CO4 3SQ, UK;gavins@ essex.ac.uk

Accepted 30 October 2012 Published Online First 24 November 2012

\section{Linked}

http://dx.doi.org/10.1136/ heartjnl-2012-303341

To cite: Sandercock GRH, Cardoso F, Almodhy M et al. Heart 2013;99: 785-790.

\title{
Cardiorespiratory fitness changes in patients receiving comprehensive outpatient cardiac rehabilitation in the UK: a multicentre study
}

\author{
Gavin R H Sandercock, ${ }^{1}$ Fernando Cardoso, ${ }^{1}$ Meshal Almodhy, ${ }^{1}$ Garyfallia Pepera ${ }^{2}$
}

\section{ABSTRACT \\ Background Exercise training is a key component of cardiac rehabilitation but there is a discrepancy between the high volume of exercise prescribed in trials comprising the evidence base and the lower volume prescribed to UK patients.}

Objective To quantify prescribed exercise volume and changes in cardiorespiratory fitness in UK cardiac rehabilitation patients.

Methods We accessed $n=950$ patients who completed cardiac rehabilitation at four UK centres and extracted clinical data and details of cardiorespiratory fitness testing pre- and post-rehabilitation. We calculated mean and effect size (d) for change in fitness at each centre and converted values to metabolic equivalent (METs). We calculated a fixed-effects estimate of change in fitness expressed as METs and $d$.

Results Patients completed 6 to 16 (median 8) supervised exercise sessions. Effect sizes for changes in fitness were $d=0.34-0.99$ in test-specific raw units and $d=0.34-0.96$ expressed as METs. The pooled fixed effect estimate for change in fitness was 0.52 METs (95\% Cl 0.51 to 0.53$)$; or an effect size of $d=0.59$ (95\% Cl 0.58 to 0.60$)$.

Conclusion Gains in fitness varied by centre and fitness assessment protocol but the overall increase in fitness (0.52 METs) was only a third the mean estimate reported in a recent systematic review (1.55 METs). The starkest difference in clinical practice in the UK centres we sampled and the trials which comprise the evidencebase for cardiac rehabilitation was the small volume of exercise completed by UK patients. The exercise training volume prescribed was also only a third that reported in most international studies. If representative of UK services, these low training volumes and small increases in cardiorespiratory fitness may partially explain the reported inefficacy of UK cardiac rehabilitation to reduce patient mortality and morbidity.

\section{INTRODUCTION}

Current UK national service guidelines for cardiac rehabilitation $^{1} 2$ cite evidence from systematic reviews ${ }^{3-5}$ of randomised controlled trials showing a $\sim 20 \%$ reduction in mortality for patients who complete exercise-based cardiac rehabilitation. The trials from which this figure was synthesised were largely completed 20-30 years ago and represent a historical vision of modern cardiac rehabilitation, ${ }^{6}$ a version particularly unrepresentative of current pharmacological practice. ${ }^{6}$ A more recent meta-analysis ${ }^{7}$ provided a more conservative estimate of the effectiveness of cardiac rehabilitation to reduce mortality and rejected its efficacy in reducing secondary cardiac events. The exact studies included within each of the numerous systematic reviews published vary due to inclusion/exclusion criteria, but common to all these reviews is the paucity of UK data. The UK's contribution to the evidence-base is two studies, both completed $>20$ years ago. ${ }^{8}$ Neither significantly reduced the risk of either total mortality or cardiac mortality in patients receiving cardiac rehabilitation compared with the usual practice group.

The recent publication of data from the RAMIT group $^{6}$ has posed some serious doubts as to the efficacy of the UK cardiac rehabilitation programme's ability to reduce patient mortality ${ }^{10}$ and morbidity or increase quality of life. It has been suggested that data from RAMIT are already outdated and do not fully represent current practice guidelines. ${ }^{11}$ Nevertheless, RAMIT still represents the most detailed randomised controlled trial of comprehensive cardiac rehabilitation in the UK. The authors suggest that the decline in the apparent effectiveness of cardiac rehabilitation is due to improved medical management (that received by intervention as well as usual practice).

Historical data suggest exercise training is an effective and fundamental element of cardiac rehabilitation (CR), ${ }^{4}$ and more recent large-scale trials of psychological interventions alone report poor efficacy of such approaches when used in isolation. ${ }^{12} 13$ A primary outcome of any exercise programme is the measurement of individuals' response to training such as changes in strength, or, as more typically reported in cardiac rehabilitation trials, cardiovascular fitness.

A $1 \%$ increase in $\mathrm{Vo}_{2 \text { peak }}$ is associated with a $2 \%$ reduction in mortality ${ }^{14}$; each 1 metabolic equivalent (MET) increase in fitness may infer a $12 \%$ decrease in mortality. ${ }^{15}$ Increases in fitness are independently associated with reduced mortality and morbidity and improved quality of life. ${ }^{16}$ Individuals who have good levels of fitness are significantly less likely to suffer cardiovascular disease. ${ }^{17-19}$ Our recent systematic review suggested cardiac rehabilitation may produce a 1.55 (95\% CI 1.22 to 1.89 ) MET increase in fitness. ${ }^{20}$ There is a relative paucity of data on changes in cardiorespiratory fitness in UK cardiac rehabilitation patients; we were not able to include any in our meta-analysis.

A fundamental difference between UK cardiac rehabilitation services and the practice reported in 
many international trials which make up the body of evidence supporting the efficacy of cardiac rehabilitation is the exercise 'dose'. In general, a greater volume of training is associated with greater improvements in fitness, ${ }^{20}$ but UK cardiac rehabilitation patients are typically only prescribed around one third the volume of exercise training ${ }^{21}$ typically received by patients in the USA or Europe. ${ }^{20}$

Along with changes in clinical practice improving survival of 'control' patients, it may simply be that UK cardiac rehabilitation does not elicit a large enough training response to provide secondary prevention of cardiovascular disease. The RAMIT study ${ }^{6}$ was detailed, but provided no information on changes in patient fitness due to cardiac rehabilitation. Given the paucity of such data, the aim of this study was to quantify the changes in cardiorespiratory fitness in a large, contemporary, multicentre sample of UK cardiac rehabilitation patients.

\section{METHODS}

\section{Study design and service characteristics}

Cardiac rehabilitation services were recruited through an email invitation sent by the British Association of Cardiovascular Prevention and Rehabilitation (BACPR) via their mailing list of UK centres. Inclusion criteria were that the cardiac rehabilitation service routinely performed cardiorespiratory fitness testing preand post-cardiac rehabilitation and that their clinical practice conformed to the recommendations of the then BACPR. ${ }^{22}$ This organisation, now the BACR, has recently updated these recommendations. ${ }^{23}$ Multicentre study ethical approval was obtained via the Integrated Research Application Service, and permission to access specified parts of records without patients' consent was obtained via the National Information Governance Bureau. However, local ethical approval could still not be obtained in two centres. Of the six services which originally volunteered, the study was completed in only four.

Data were obtained via retrospective analysis of patient records obtained during supervised outpatient cardiac rehabilitation. Patients' age, sex, body mass and primary diagnosis (reason for attending CR) were recorded from individual records. Patients' reason for attending cardiac rehabilitation was classified as being due to myocardial infarction (post-MI), having received elective revascularisation therapy including coronary artery bypass grafting (CABG), percutaneous coronary intervention (PCI) or other (angina pectoris, valvuloplasty). We did not include patients with congestive heart failure. We also recorded the type of test used and individual performance on the test at both pre- and post-CR. The metric used to describe performance depended on the test performed.

At the level of the service, we recorded the types of exercise test used and reasons for test selection. We also recorded the average duration (weeks), frequency (sessions per week) and type of exercise training used in the rehabilitation programme. All services included a core component of supervised exercise training and some aspect of formal patient education or counselling on lifestyle change. All services used mixed circuit-based (aerobic and resistance) exercise training sessions of 60 min duration, including a 15 min warm-up and cool-down performed at beginning and end. ${ }^{1}$ The characteristics of the four services evaluated are given in table 1 and are broadly comparable with national survey data. ${ }^{21}$

\section{Data extraction and treatment}

The cardiac rehabilitation services used four different exercise tests: the Bruce treadmill protocol, the 6-min walk test (6MWT), the incremental shuttle walking test (ISWT), and an incremental cycle ergometer test. The data extracted from records to compare these are briefly described below; pre- and post-cardiac rehabilitation tests were treated identically in all cases. The Bruce treadmill protocol is a standardised incremental treadmill test with 3-min stages. Time completed on the test was recorded as a measure of test performance. Time was converted first to $\mathrm{Vo}_{2 \text { peak }}(\mathrm{ml} / \mathrm{kg} / \mathrm{min})$ and then to METs. One centre used an incremental cycling test using an electronicallybraked cycle ergometer. The test comprised 2-min stages of increasing speed which was titrated at individual level based on sex, body size and self-reported fitness and physical activity. Final work-rate (Watts) was recorded as a measure of test performance for each patient and converted to METs based on standard equations. ${ }^{24}$ The $6 \mathrm{MWT}$ is a self-paced field test of functional capacity most commonly used in patients with heart failure. The total distance walked by patients in 6 min was recorded as a measure of test performance and converted to average walking speed, then METs. ${ }^{24}$ The ISWT is currently recommended for use in cardiac rehabilitation patients. ${ }^{1}$ Distance walked by each patient was recorded as a measure of test performance, then converted to $\mathrm{VO}_{2 \text { peak }}(\mathrm{ml} / \mathrm{kg} / \mathrm{min})^{25}$ and then to METs for comparability with other tests.

\section{Statistical analysis}

Each of the tests used reported performance in different units. For each test, we calculated the pre- versus post-test difference in performance measures and reported mean change with $95 \%$ CI for the given units. ${ }^{26} 27$ We also reported change in performance in METs and as standardised effect size (Cohen's d) as these are both readily understood and make our findings easily comparable with those of other studies. Finally, we calculated a fixed-effects pooled estimate of change in performance preversus post-cardiac rehabilitation expressed as METs and effect size (d).

\section{RESULTS}

The descriptive characteristics of the different patient groups within the four cardiac rehabilitation centres are given in table 1. There were differences in routine testing practice between centres. Centre A used the Bruce treadmill protocol to assess post-MI and valve replacement patients, but used the ISWT to assess revascularisation patients. Centre A used the 6MWT to assess a more heterogeneous group of patients, mainly those receiving valvuloplasty. Centre $\mathrm{B}$ used a mixture of cycle ergometry and ISWT to assess all patients eligible for cardiac rehabilitation (choice of test was dependent on space and availability of equipment). Centres C and D exclusively used the ISWT in all patients. Patients in centre $\mathrm{B}$ were equally likely to be attending cardiac rehabilitation following elective revascularisation or MI, but the majority of patients in centres C and D were attending cardiac rehabilitation following elective revascularisation. Table 2 shows the changes in cardiorespiratory fitness test performance in each group of patients in each cardiac rehabilitation centre. Patients in centre A showed a large improvement $(\mathrm{d}=0.85)$ in time spent on the Bruce treadmill protocol, corresponding to an increase of 0.76 METs. The mixed patient group from centre B, assessed by cycle ergometry, showed a moderate increase in cardiorespiratory fitness, expressed as peak volitional work-rate achieved (W) $(\mathrm{d}=0.57)$; equivalent to an increase of 0.50 METs. Change in distance walked on the $6 \mathrm{MWT}$ in the patients receiving elective revascularisation and valvuloplasty (centre A) was small $(\mathrm{d}=0.34)$, equivalent to only 0.33 METs. 
Table 1 Sample characteristics by exercise test groups and basic exercise prescription data at each centre for all patients ( $\mathrm{n}=950$ )

\begin{tabular}{|c|c|c|c|c|c|c|c|c|}
\hline Centre & Patient groups and exercise tests & Sample size, $\mathbf{n}$ & Dates & $\begin{array}{l}\text { Mean (SD) } \\
\text { age (years) }\end{array}$ & Sex & Primary diagnoses & $\begin{array}{l}\text { Programme } \\
\text { duration (weeks) }\end{array}$ & $\begin{array}{l}\text { Weekly exercise } \\
\text { sessions }\end{array}$ \\
\hline \multirow[t]{3}{*}{ A } & i. Bruce treadmill test & 125 & 2006-10 & $64.1(10.2)$ & $\begin{array}{l}\text { Male: } 73 \% \\
\text { Female: } 27 \%\end{array}$ & $\begin{array}{l}\text { Post-MI: } 76 \% \\
\text { Other: } 34 \%\end{array}$ & 8 & 1 \\
\hline & ii. ISWT & 104 & 2006-10 & $67.5(10.2)$ & $\begin{array}{l}\text { Male: } 82 \% \\
\text { Female: } 18 \%\end{array}$ & $\begin{array}{l}\text { Post-Ml: } 2 \% \\
\text { Revasc: } 76 \% \\
\text { Other: } 12 \%\end{array}$ & & 1 \\
\hline & iii. 6-min walk test & 54 & $2006-10$ & $69.4(7.7)$ & $\begin{array}{l}\text { Male: } 73 \% \\
\text { Female: } 27 \%\end{array}$ & $\begin{array}{l}\text { Post-MI: } 37 \% \\
\text { Revasc: } 22 \% \\
\text { Other: } 41 \%\end{array}$ & & 1 \\
\hline B & Incremental cycle ergometry and ISWT & 221 & $2009-11$ & $69.5(9.7)$ & $\begin{array}{l}\text { Male: } 73 \% \\
\text { Female: } 27 \%\end{array}$ & $\begin{array}{l}\text { Post-MI } 40 \% \\
\text { Revasc: } 42 \% \\
\text { Other: } 18 \%\end{array}$ & 8 & 1 or 2 \\
\hline C & ISWT & 81 & 2007-10 & $57(11)$ & $\begin{array}{l}\text { Male: } 85 \% \\
\text { Female: } 15 \%\end{array}$ & $\begin{array}{l}\text { Post-MI: } 25 \% \\
\text { Revasc:71\% } \\
\text { Other: } 4 \%\end{array}$ & 6 & 1 or 2 \\
\hline D & ISWT & 365 & $2001-10$ & $62.9(15.2)$ & $\begin{array}{l}\text { Male: } 73 \% \\
\text { Female: } 27 \%\end{array}$ & $\begin{array}{l}\text { Post-MI: } 34 \% \\
\text { Revasc: } 64 \% \\
\text { Other: } 2 \%\end{array}$ & 8 & 1 \\
\hline
\end{tabular}

ISWT, incremental shuttle walking test; Post-MI, post-myocardial infarction; Revasc, post-elective revascularisation therapy (CABG or PCI); Other, patients receiving cardiac rehabilitation for other treatments and conditions (angina pectoris, valvuloplasty recipients, resynchronisation therapy).

All centres reported using the ISWT in at least some patients; in two centres (C and D) this was the only test routinely used and was employed in the assessment of all patients who could complete it. The predominantly elective revascularisation patients (centre A) improved their test performance by a mean of $160 \mathrm{~m}$, producing a large effect size $(\mathrm{d}=0.99)$. Patients from mixed MI and revascularisation groups (centres B, C, D) showed relatively homogenous changes in ISWT performance,

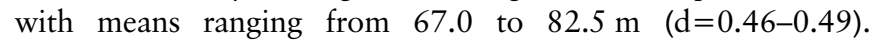
Converting these changes in distance to METs, patients from centre A improved their fitness by an average of 0.70 METs which resulted in a larger effect size $(d=0.96)$ than patients from centres B, C and D, who improved by $0.40,0.37$ and 0.51 METs respectively (effect sizes: $\mathrm{d}=0.57, \mathrm{~d}=0.46$ and $\mathrm{d}=0.58$ ).

The pooled fixed effect estimate for mean change in cardiorespiratory fitness in all $(\mathrm{n}=950)$ patients was $0.52(95 \% \mathrm{CI}$ 0.51 to 0.53 ) METs. This was equivalent to a moderate overall effect size for change in fitness of $d=0.59$ (95\% CI 0.58 to $0.60)$.

\section{DISCUSSION}

The aim of this study was to quantify changes in cardiorespiratory fitness in patients receiving cardiac rehabilitation in the UK.
The results of this study suggest that the supervised exercise training prescribed as part of UK cardiac rehabilitation does not produce changes in cardiorespiratory fitness comparable with international studies. It also appears that changes in fitness vary greatly between UK cardiac rehabilitation centres, all of which adhere to the same practice guidelines, and that the assessment protocols used to measure change in fitness may account for at least some of this heterogeneity.

Compared with a recent meta-analysis of international studies, ${ }^{20}$ which reported a mean increase of 1.55 METs, the overall increase reported here $(0.52 \mathrm{METs})$ is very conservative. The meta-analysis was based on treadmill test data, the gold standard assessment for cardiorespiratory fitness, and concluded that the treadmill test protocol was a significant source of the between-trial heterogeneity. There are very few comparable UK studies of change in fitness due to cardiac rehabilitation, but survey data suggest this gold standard measure is rarely used in routine assessment of patients both pre- and post-cardiac rehabilitation. One study, ${ }^{28}$ which was not included in the recent meta-analysis due to omissions in data reporting, demonstrated a $90 \mathrm{~s}$ increase in treadmill time for CABG patients assessed using the Bruce protocol; the same protocol used to assess as post-MI patients in centre A. This is equivalent to an

Table 2 Changes in cardiorespiratory fitness test performance in original units and METs pre- vs post-cardiac rehabilitation (CR)

\begin{tabular}{|c|c|c|c|c|c|c|c|c|c|}
\hline Test* & $\begin{array}{l}\text { Sample } \\
\text { size }\end{array}$ & Pre-CR & Post-CR & Change $(95 \% \mathrm{Cl})$ & $\begin{array}{l}\text { Effect } \\
\text { size (d) }\end{array}$ & Pre (METs) & Post (METs) & Change $(95 \% \mathrm{Cl})$ & $\begin{array}{l}\text { Effect size } \\
\text { (d) }\end{array}$ \\
\hline${ }^{\text {aBruce treadmill test }(s)}$ & $n=125$ & $329(132)$ & $451(153)$ & 121 (81 to 140$)$ & 0.85 & $6.8(2.1)$ & $7.5(2.4)$ & $0.76(0.40$ to 1.12$)$ & 0.58 \\
\hline aIncremental shuttle test (m) & $\mathrm{n}=104$ & $295(132)$ & $455(190)$ & 160.1 (136.2 to 184.1$)$ & 0.99 & $4.4(0.59)$ & $5.1(0.85)$ & $0.70(0.58$ to 0.86$)$ & 0.96 \\
\hline${ }^{\mathrm{a}} 6$-min walk test $(\mathrm{m})$ & $\mathrm{n}=54$ & $279(144)$ & $329(148)$ & 50.3 (18.8 to 81.6$)$ & 0.34 & $3.2(0.97)$ & $3.6(0.99)$ & $0.33(0.13$ to 0.54$)$ & 0.34 \\
\hline${ }^{b}$ Incremental cycle ergometry (W) & $n=103$ & $87.6(19.1)$ & $98.5(19.1)$ & 10.9 (8.6 to 12.2$)$ & 0.57 & $5.6(0.8)$ & $6.0(0.8)$ & $0.40(0.31$ to 0.51$)$ & 0.55 \\
\hline${ }^{\mathrm{b}}$ Incremental shuttle test (m) & $\mathrm{n}=118$ & 295 (139) & $362(155)$ & 67.0 (51.6 to 83.6$)$ & 0.46 & $4.6(0.7)$ & $5.0(0.7)$ & $0.40(0.30$ to 0.52$)$ & 0.57 \\
\hline Incremental shuttle test $(\mathrm{m})$ & $n=81$ & $420(157)$ & $503(179)$ & 82.5 (53.9 to 111.3$)$ & 0.49 & $5.2(0.76)$ & $5.5(0.84)$ & $0.37(0.25$ to 0.48$)$ & 0.46 \\
\hline${ }^{\mathrm{d}}$ Incremental shuttle test $(\mathrm{m})$ & $n=365$ & $341(165)$ & $411(230)$ & 71.5 (57.6 to 85.4$)$ & 0.46 & $4.9(0.83)$ & $5.4(0.94)$ & $0.51(0.46$ to 0.56$)$ & 0.58 \\
\hline Pooled fixed effect model: & $\mathrm{n}=950$ & & & & & & & $0.52(0.51$ to 0.53$)$ & $\begin{array}{l}0.59(95 \% \mathrm{Cl} \\
0.58 \text { to } 0.60)\end{array}$ \\
\hline
\end{tabular}

*Superscripts a-d designate the four centres which participated.

Effect size (d), Cohen's d; MET, metabolic equivalent; Post-CR, exercise test at exit from phase III cardiac rehabilitation; Pre-CR, exercise test at entry to phase III cardiac rehabilitation. 
increase of approximately 0.60 METs, which is less than the value reported here. This was despite patients receiving 16 supervised exercise sessions compared with only eight in the present study. One reason for the larger increase in fitness observed in the present study may be differences in the clinical characteristics of the patient groups. Post-MI patients increased their fitness by more than elective revascularisation patients in our recent meta-analysis $(\mathrm{d}=2.08$ vs $\mathrm{d}=0.97)$. Both these effect sizes are well in excess of that reported presently $(\mathrm{d}=0.77)$, but our estimate is actually quite similar to that which we previously reported for change in studies using the Bruce treadmill protocol $(d=0.79)$. The Bruce protocol is characterised by large increments $^{29}$ and may not be the most suitable protocol for the assessment of older patients, such as cardiac rehabilitation recipients who often present with orthopaedic co-morbidities.

Treadmill testing is also costly and time consuming, which has lead to many UK cardiac rehabilitation centres employing fieldbased estimates of cardiorespiratory fitness. There are relatively few data regarding the value of incremental cycle ergometry in assessing cardiac rehabilitation patients. Exercise test performance during cycle ergometry appears to be sensitive to changes in fitness due to cardiac rehabilitation and can provide important prognostic information. ${ }^{30} \mathrm{Di}$ Valentino et $a l^{30}$ reported differences of $148( \pm 47)$ versus $124( \pm 38)$ W between surviving and non-surviving cardiac rehabilitation patients. They also reported an improvement in work capacity of $24 \mathrm{~W}$; more than twice that observed in the patients from centre B $(10.4 \mathrm{~W})$. Differences in the test protocol may account for some of the variation in improvement, but the very large differences in exercise training seem more likely. Di Valentino et $a l^{30}$ describe in detail the exercise training protocol in this German rehabilitation programme as comprising a 4-week build-up phase with daily rehabilitation activities taking up to $3 \mathrm{~h}$ per day, then a consolidation phase of 8 weeks, exercising $3 \times 2 \mathrm{~h}$ per week. The total exercise dose over 12 weeks was, therefore, $132 \mathrm{~h}$ (84 intense plus $48 \mathrm{~h}$ consolidation). In comparison, patients at centre B received either 1- or 2-hourly sessions over a maximum of 8 weeks, equating to only $8-16$ h total exercise.

The 6MWT is commonly used in the assessment of patients with heart failure, but few data are available in typical cardiac rehabilitation patients ${ }^{31}$ and we found no data comparable to those for changes in fitness due to exercise programmes like those reported here. Even in patients with heart failure, the 6MWT does not appear to be as sensitive to changes in fitness as the ISWT ${ }^{32}$; this lack of sensitivity may explain the small effect size for change in test performance observed here.

The most commonly used field test in UK cardiac rehabilitation centres is the ISWT, ${ }^{21}$ and a number of studies have reported changes in ISWT performance of $60-100 \mathrm{~m}$ due to cardiac rehabilitation. ${ }^{33-36}$ Little has been reported on the potential dose-response between exercise and changes in fitness test performance using this protocol. Arnold et $a l^{37}$ recently reported no difference in a sample of cardiac patients exercising once or twice per week, but their results are difficult to interpret due to differences in test performance at baseline. ${ }^{38} \mathrm{~A}$ more recent study in patients with very similar baseline scores also reported no differences in training response of patients exercising either once or twice per week. ${ }^{38}$ Of interest, this study is one of the few to report ISWT performance in METs as opposed to the distance walked during the test. This detail may be of some importance and deserves elucidation.

The ISWT, as the name implies, is an incremental test in which the patient is required to walk faster during each sequential stage. The use of distance to describe fitness test performance is clearly useful in the clinical setting. Distance is a metric readily understood by practitioners and patients and changes in shuttle walking tests can be easily monitored and expressed as change in $\mathrm{m}$. The magnitude of change in walking distance is commonly reported to be around $100 \mathrm{~m}$ in patients receiving outpatient cardiac rehabilitation. ${ }^{33-38}$ Cardiorespiratory fitness itself, however, represents the ability to produce and maintain a given work rate, not a work capacity. From an exercise physiology perspective therefore, it would be more correct to express ISWT performance as walking speed $(\mathrm{m} / \mathrm{s}$ or $\mathrm{km} / \mathrm{h})$, or better still, as estimated $\mathrm{VO}_{2 \text { peak }}(\mathrm{ml} / \mathrm{kg} / \mathrm{min})^{25}$ or METs. This practice is uncommon within the scientific literature and to our knowledge only two studies have reported such metrics. ${ }^{38} 39$ There are additional drawbacks with the use of distance as an expression of an individual's fitness. While study results are often highly statistically significant $(p<0.01)$, there is little evidence of power analyses in any of the published data. Due to this, such changes have been interpreted as clinically significant despite no clinically meaningful lower cut-off being available. Like any fixed stage time incremental protocol, there are many more shuttles per level in the latter stages of the test than there are early on. This means that changes in distance walked in excess of $100 \mathrm{~m}$ can be attained without the need for and individual to increase walking speed (work rate). Changes in walking speed (and therefore estimated METs) are therefore commonly much smaller than changes in distance walked, with many patients not increasing work rate (fitness) at all. In the quest for easily interpretable values and statistical significance, it appears that some authors have neglected to assess whether the changes in distance walked during the ISWT represent a clinically meaningful improvement in cardiorespiratory fitness.

Our data suggest that patients attending UK cardiac rehabilitation centres can only expect a 0.59 MET increase in their cardiorespiratory fitness over a typical $6-8$-week programme. This value may be slightly smaller if their fitness is assessed using the ISWT (0.54 METs). These values represent approximately one third the improvement in fitness reported in the literature. ${ }^{20}$ While testing modality may account for some of this difference, it is of interest that even in the group who improved most in the present study (post-MI patients tested on a treadmill), the magnitude of change was less than half the value synthesised from international studies.

The starkest difference between the studies analysed in the recent systematic review and the present groups is the total volume of exercise completed during outpatient cardiac rehabilitation. We previously found that total number of exercise bouts was a significant mediator of change in fitness. Using a median split of the number exercise sessions, we have previously reported greater gains in fitness for patients receiving $>36$ exercise sessions than in those receiving 36 or less. Brodie et $a l^{21}$ reported that UK patients undertake a mean of 11.6 exercise sessions. In common with the magnitude of change in fitness, the UK exercise dose is also one third that reported in the literature. ${ }^{20}$ Patients in the present study received a modal value of 8 exercise sessions (range 6-16); the lower end of this range is similar to the exercise prescription which had no significant impact on clinical outcomes in RAMIT. ${ }^{6}$

There is a clear dose-response between increases in fitness and reductions in mortality. ${ }^{14}$ Given that a 1 MET increase in fitness is needed to elicit a $12 \%$ reduction in mortality, ${ }^{15}$ it seems unsurprising therefore that the UK cardiac rehabilitation services examined in RAMIT did not significantly reduce mortality. ${ }^{6}$ 


\section{Strengths and limitations}

The strengths of the present study lie in the large sample size and the inclusive nature of the study population afforded by the retrospective design. Conversely, a major limitation is the lack of any control group. The cohort design means we cannot quantify how much of the reported improvement in fitness is due to cardiac rehabilitation per se. There is evidence of some spontaneous recovery in fitness in patients following revascularisation and $\mathrm{MI},{ }^{40}{ }^{41}$ suggesting that the current study is likely to overestimate the real effect that cardiac rehabilitation has on patients' cardiorespiratory fitness. The use of patient records allowed us to quickly gather a large sample without the bias of requiring consent to participate in a research study; it was also a limitation as there were differences in the recording methods used between centres. All centres described the testing protocols as 'symptom limited' and all included attainment of $85 \%$ peak predicted maximum heart rate as termination criteria. While not reported here, patient records indicated that volitional termination was by far the most common reason for stopping the incremental exercise tests. All centres reported that they applied the ISWT according to national guidance, ${ }^{1}$ but this was often performed by a variety of individuals (nurses, physiotherapists, exercise physiologists) within each centre. Our retrospective design does not allow us to standardise test variables such as 'encouragement' or 'patient motivation' either within or between centres.

For the purpose of this study we only included patients with complete data necessary for this analysis. This meant the rejection of $>1200$ patient records due primarily to having either incomplete or insufficient data, but also due to the patients' failure to complete the rehabilitation programme or to return for retesting.

\section{CONCLUSIONS}

Our findings suggest that the outpatient cardiac rehabilitation programmes sampled in our study commonly prescribed a dose of exercise insufficient to provide meaningful benefits to patients. The volume of exercise prescribed is equivalent to approximately one third that which a patient in North America typically receives. Many of the trials systematically reviewed to produce estimates of cardiac rehabilitation's ability to reduce mortality contain a much higher volume of exercise than typical UK services prescribe. While we cannot confirm the representativeness of our sample in terms of UK outpatient cardiac rehabilitation, these findings may offer some insight to explain the somewhat disappointing results of RAMIT. ${ }^{6}$ When increases in patient fitness are quantified, the response of the UK cardiac rehabilitation patients receiving between 6 and 16 supervised exercise sessions is much less than that reported in our systematic review of international trials in which a median of 36 sessions was prescribed. ${ }^{20}$ We suggest that cardiac rehabilitation patients in the UK are not receiving the full potential benefit available from supervised outpatient cardiac rehabilitation. As an example, if international trials of a drug demonstrated its efficacy at a daily dose of $600 \mathrm{mg}$, it seems unlikely that doctors would routinely prescribe $200 \mathrm{mg}$ to UK patients. Whether this is through ignorance of the fineries of exercise prescription, or to spatial, temporal and financial restrictions in service provision, such under-prescription of exercise seems to be common practice in UK cardiac rehabilitation.

In summary, there is clear incongruence between the evidence base for cardiac rehabilitation and current clinical practice in the UK. Clearly, further trials and systematic reviews of UK cardiac rehabilitation data are needed to confirm our findings, but it seems likely that increased funding to facilitate a greatly increased frequency and/or duration of exercise training during outpatient rehabilitation is needed. Such changes will of course produce new challenges to cardiac rehabilitation practitioners, not only financial in nature but also with regard to patients' motivation to take up and adhere to exercise training.

Contributors GRHS designed the study and wrote the manuscript. MA and GP collected, treated and analysed the data. All authors reviewed and copy-edited the manuscript prior to submission. GRHS is the guarantor for this article.

Funding None.

Competing interests None.

Ethics approval IRAS.

Provenance and peer review Not commissioned; internally peer reviewed.

\section{REFERENCES}

1 Scottish Intercollegiate Guidance Network. SIGN 57 cardiac rehabilitation. A national clinical guideline. Edinburgh: Royal College of Physicians, 2002.

2 Department of Health. National Service Framework for Coronary Heart Disease: Modern Standards and Service Models.London: The Stationery Office, 2000.

3 O'Connor GT, Buring JE, Yusuf $S$, et al. An overview of randomized trials of rehabilitation with exercise after myocardial infarction. Circulation 1989;80:234-44.

4 Taylor RS, Brown A, Ebrahim S, et al. Exercise-based rehabilitation for patients with coronary heart disease: systematic review and meta-analysis of randomized controlled trials. Am J Med 2004;116:682-92.

5 Jolliffe JA, Rees K, Taylor RS, et al. Exercise-based rehabilitation for coronary heart disease. Cochrane Database Syst Rev 2001;CD001800.

6 West RR, Jones DA, Henderson AH. Rehabilitation after myocardial infarction trial (RAMIT): multi-centre randomised controlled trial of comprehensive cardiac rehabilitation in patients following acute myocardial infarction. Heart 2012;98:637-44.

7 Heran BS, Chen JMH, Ebrahim S, et al. Exercise-based cardiac rehabilitation for coronary heart disease. Cochrane Database of Systematic Reviews 2011.

8 Bethell HJ, Mullee MA. A controlled trial of community based coronary rehabilitation. Br Heart J 1990;64:370-5.

9 Carson $\mathrm{P}$, Phillips R, Lloyd M, et al. Exercise after myocardial infarction: a controlled trial. J R Coll Physicians Lond 1982;16:147-51.

10 Wood D. Is cardiac rehabilitation fit for purpose in the NHS: maybe not. Heart 2012;98:607-8.

11 Members BEC. RAMIT presents an outdated version of cardiac rehabilitation. Heart 2012;98:672; author reply 3-4.

12 Jones DA, West RR. Psychological rehabilitation after myocardial infarction: multicentre randomised controlled trial. BMJ 1996;313:1517-21.

13 Berkman LF, Blumenthal J, Burg M, et al. Effects of treating depression and low-perceived social support on clinical events after myocardial infarction-The enhancing recovery in coronary heart disease patients (ENRICHD) randomized trial. Jama-J Am Med Assoc 2003;289:3106-6.

14 Vanhees $L$, Fagard R, Thijs $L$, et al. Prognostic value of training-induced change in peak exercise capacity in patients with myocardial infarcts and patients with coronary bypass surgery. Am J Cardiol 1995;76:1014-19.

15 Myers J, Prakash M, Froelicher V, et al. Exercise capacity and mortality among men referred for exercise testing. N Engl J Med 2002;346:793-801.

16 Lavie CJ, Milani RV. Benefits of cardiac rehabilitation and exercise training in elderly women. Am J Cardiol 1997;79:664-6.

17 McAuley P, Pittsley J, Myers J, et al. Fitness and fatness as mortality predictors in healthy older men: the veterans exercise testing study. J Gerontol A Biol Sci Med Sci 2009;64:695-9.

18 Lyerly GW, Sui $X$, Church TS, et al. Maximal exercise electrocardiography responses and coronary heart disease mortality among men with diabetes mellitus. Circulation 2008; 117:2734-42.

19 Sui X, Laditka JN, Hardin JW, et al. Estimated functional capacity predicts mortality in older adults. J Am Geriatr Soc 2007;55:1940-7.

20 Sandercock G, Hurtado V, Cardoso F. Changes in cardiorespiratory fitness in cardiac rehabilitation patients: A meta-analysis. Int J Cardiol 2011. doi: http://dx.doi.org/10. 1016/j.bbr.2011.03.031

21 Brodie D, Bethell H, Breen S. Cardiac rehabilitation in England: a detailed national survey. Eur J Cardiovasc Prev Rehabil 2006;13:122-8.

22 BACR. Standards and Core Components for Cardiac Rehabilitation (2007). 2007.

23 BACPR. The BACPR Standards and Core Components for Cardiovascular Disease Prevention and Rehabilitation 2012 (2nd Edition). 2012.

24 ACSM. ACSM's guidelines for exercise testing and prescription. Philadelphia: Lippincott Williams \& Wilkins, 2010. 
25 Fowler SJ, Singh S. Reproducibility and validity of the incremental shuttle walking test in patients following coronary artery bypass surgery. Physiotherapy 2005;91:22-7.

26 Hopkins WG, Marshall SW, Batterham AM, et al. Progressive statistics for studies in sports medicine and exercise science. Med Sci Sports Exerc 2009;41:3-13.

27 Batterham AM, Hopkins WG. Making meaningful inferences about magnitudes. International journal of Sports Physiology and Performance 2006;1:50-7.

28 Sharma A, McLeod AA. Cardiac rehabilitation after coronary artery bypass graft surgery: its effect on ishcaemia, functional capacity and a mulitvariate index of prognosis. Coronary Health Care 2001;5:189-93.

29 Bruce RA, Blackmon JR, Jones JW, et al. Exercising Testing in Adult Normal Subjects and Cardiac Patients. Pediatrics 1963;32(Suppl):742-56.

30 Di Valentino M, Maeder MT, Jaggi S, et al. Prognostic value of cycle exercise testing prior to and after outpatient cardiac rehabilitation. Int J Cardiol 2010;140:34-41.

31 Opasich C, De Feo S, Pinna GD, et al. Distance walked in the 6-minute test soon after cardiac surgery: toward an efficient use in the individual patient. Chest 2004:126:1796-801.

32 Morales FJ, Montemayor T, Martinez A. Shuttle versus six-minute walk test in the prediction of outcome in chronic heart failure. Int J Cardiol 2000;76:101-5

33 Tobin D, Thow MK. The $10 \mathrm{~m}$ Shuttle Walk Test with Holter Monitoring: an objective outcome measure for cardiac rehabilitation. Coronary Health Care 1999;3:3-17.
34 Fowler S, Singh S, Revill S. Reproducibility and validity of the incremental shuttle walking test in patients following coronary artery bypass surgery. Physiotherapy 2005:91:22-7.

35 Sandercock GR, Grocott-Mason R, Brodie DA. Changes in short-term measures of heart rate variability after eight weeks of cardiac rehabilitation. Clin Auton Res 2007;17:39-45

36 Arnott AS. Assessment of functional capacity in cardiac rehabilitation. Coronary Health Care 1997;1:30-6.

37 Arnold HJ, Sewell L, Singh SJ. A comparison of once- versus twice-weekly supervised phase III cardiac rehabilitation. Br J Cardiol 2007;14:45-8.

38 Almodhy MY, Sandercock GR, Richards L. Changes in cardiorespiratory fitness in patients receiving supervised outpatient cardiac rehabilitation either once or twice a week. Int J Cardiol 2012;160:215-6.

39 Pepera G, McAllister J, Sandercock G. Long-term reliability of the incremental shuttle walking test in clinically stable cardiovascular disease patients. Physiotherapy 2010;96:222-7.

40 Dressendorfer RH, Franklin BA, Cameron JL, et al. Exercise training frequency in early post-infarction cardiac rehabilitation. Influence on aerobic conditioning. J Cardiopulm Rehabil 1995;15:269-76.

41 Sivarajan ES, Bruce RA, Lindskog BD, et al. Treadmill test responses to an early exercise program after myocardial infarction: a randomized study. Circulation $1982 ; 65: 1420-8$ 